\title{
Formación docente en la sociedad del conocimiento
}

\section{INÉS AGUERRONDO*}

\section{RESUMO}

O presente artigo debate a urgência de um novo modelo de formação docente baseado na inovação. A proposta vigente necessita de um salto de perspectiva que supere as lógicas clássicas de instituições fechadas e garanta a preparação de novos docentes para novas escolas, no marco de um novo sistema educativo, cujo compromisso seja a formaçãopara-a-ação. O texto, refletindo sobre cinco dimensões fundamentais da prática docente (a pedagógico-didática, a político-institucional, a produtiva, a interativa e a especificadora), propõe dez ideias-força que servem de base para um novo modelo de formação inicial e contínua dos docentes.

Palavras-chave: Formação de professores, Inovação pedagógica, Prática de ensino, Aprendizagem.

\section{RESUMEN}

El presente artículo debate sobre la urgencia de un nuevo modelo de formación docente basado en la innovación. La propuesta vigente necesita un cambio de perspectiva que supere las lógicas clásicas de instituciones cerradas y que garantice la preparación de nuevos docentes para nuevas escuelas, en el marco de un nuevo sistema educativo, cuyo compromiso sea la formación para la acción. El texto reflexiona sobre cinco dimensiones fundamentales de la práctica docente (la pedagógico-didáctica, la político-institucional, la

\footnotetext{
* Coordinadora del Área Gestión del Departamento de Educación (Universidad Católica Argentina) y profesora de la escuela de Educación de la Universidad de San Andrés (Argentina). Forma parte del personal de Unesco/ IIPE, sede Buenos Aires (inesague@gmail.com).
} 
productiva, la interactiva y la especificadora) y propone diez ideas-fuerza que sirven de base para un nuevo modelo de formación inicial y continua de los docentes.

Palabras clave: Formación docente; Innovación pedagógica; Práctica de la enseñanza, Aprendizaje.

\section{Teacher training in the knowledge society}

\section{ABSTRACT}

This article discusses the urgency of a new model of teacher training based on innovation. The current proposal requires a leap of perspective that will go beyond the logic of classical closed institutions and ensure the preparation of new teachers for new schools, as part of a new educational system, whose commitment is training-for-action. This article, reflecting on five key dimensions of teaching practice (pedagogical-didactic, political-institutional, productive, interactive and specifying), proposes ten key ideas that underpin a new model for the initial and continuing education of teachers.

Keywords: Teacher education, Educational innovation, Teaching practice, Learning.

"Apenas podemos imaginar las demandas y posibilidades que enfrentará la educación en el siglo 21. Pero todo indica que ella experimentará un verdadero cambio de marea. Es decir, transformaciones que no pertenecen a un orden trivial ni acumulativo...Posiblemente estemos a las puertas de una cuarta revolución..., impulsada por rápidos y decisivos cambios en el entorno dentro del cual se organiza la educación y de las teorías y conceptos que rigen su producción."

(Brunner, 2000)

\section{LA ERA DE LA INNOVACIÓN}

En solo unas décadas la sociedad industrial dejó paso a la sociedad de la información, y esta a la sociedad del conocimiento. Un dato semejante debiera impactar, y de hecho impacta, en los sistemas educativos. Todos ellos están intentando cambiar, pero están en difícil situación 
debido a que la vieja estrategia de ajuste a las demandas sociales por expansión (más alumnos, más escuelas, más docentes) ya no alcanza. Hoy los cambios que se requieren no son cuantitativos sino que deben encararse cambios cualitativos. Los viejos modelos, que fueron un indudable avance en su momento, han demostrado suficientemente que ya no son la respuesta. Han dejado, sin embargo, su secuela de problemas estructurales insolubles y, a la luz del pensamiento actual, están demostrando su ineficacia para inventar las soluciones adecuadas.

El problema es especialmente complicado porque la rapidez del cambio hace que el objetivo sea una especie de "blanco móvil". Ya no basta diseñar un sistema educativo más moderno que el que tenemos y llevarlo a la realidad porque lo cierto es que cuando se concrete ya será obsoleto. A diferencia de las anteriores, las actuales propuestas de cambio educativo deben incorporar como novedad el dato del cambio constante. Se necesita inventar nuevos modelos de oferta educativa que contengan, en su mismo diseño, los mecanismos adecuados para actualizarse permanentemente.

La inadecuación de las estrategias probadas aparece claramente a la luz de los procesos de reforma educativa de la última mitad del siglo XX. En ese lapso se sucedieron diferentes estrategias de cambio educativo, desde las iniciales políticas "reactivas" de los años 60 hasta las concepciones de cambio "programático" que empezaron a desarrollarse en la década de los 80 (Aguerrondo, 1983). La diferencia entre estos dos tipos de estrategia reside en que en un caso se dan respuestas de mero ajuste a las demandas sociales (que en general se refieren a cambios poco profundos y a más cantidad de educación) y en el otro se intenta mejorar el sentido y la calidad de la oferta. Muchos autores hablan por esto de varias olas de reformas educativas (Gajardo, 1999; Brunner, 2000) que se diferencian por las concepciones básicas que las sustentan.

La crisis del Estado de Bienestar impacta también en estos procesos. A partir de los 80 se retoma con fuerza el problema de la modernización del Estado que se suma como problemática a la necesidad de cambio educativo. Para analizar las reformas educativas se introducen también los conceptos de reformas de primera y segunda generación (Oszlak, 1999). Las reformas de primera generación son reformas "hacia fuera", es decir que tratan de aligerar el compromiso central del Estado, lo que en el campo de la educación da origen a la controvertida cuestión de la descentralización, el financiamiento de la demanda, la privatización, etc. Las reformas de segunda generación, son reformas "hacia adentro", institucionales, que suponen una real transformación, lo que implica pasar a una nueva forma de hacer socialmente educación (Aguerrondo, 2001). 
Las propuestas de cambio educativo, para ser viables en términos de futuro, deben ser cualitativamente diferentes de las que se han implementado hasta ahora. Porque "las reformas de segunda generación suponen, por su propia naturaleza, una concepción distinta de la modernización. La clave, de ahora en adelante, será generar organizaciones escolares capaces, ellas mismas, de aprender. Si los profesores, las escuelas y los sistemas en su conjunto no desarrollan la capacidad de aprender de los éxitos y fracasos de la experiencia pasada, los problemas que se resuelven hoy reaparecerán mañana. Por eso, si los beneficios de la reforma educacional han de perdurarse, los profesores deben aprender a llevar a cabo su propia investigación-acción para identificar problemas y buscar soluciones; los supervisores deben desempeñar un rol en facilitar ese tipo de investigación y los formadores de los docentes deben simultáneamente apoyarlos y comunicar a los futuros maestros las lecciones aprendidas" (Brunner, 2000).

Se trata de lograr salas de clase donde se desarrollen no solo saberes complejos sino básicamente competencias en los alumnos, que implican no solamente el conocimiento sino la capacidad de actuar de manera adecuada para participar concientemente de la vida personal y social. Esto no puede hacerse desde el modelo 'frontal' de comunicación educativa sobre el cual se estructuraron tradicionalmente los sistemas escolares (Shiefelbein, 1992). Se requiere poder inventar escuelas flexibles capaces de poder variar indefinidamente, de acuerdo con las necesidades, sus tiempos, espacios, agrupamientos, superando los modelos rígidos y homogéneos incapaces de soportar la diversidad. Lugares de aprendizaje que tengan la posibilidad de adaptarse de manera permanente a la cambiante situación dinámica de hoy.

Un cambio de este tipo presenta una dificultad adicional referida a como reconocerlo. "Las innovaciones educacionales nacen menos de un plan que de una manera distinta de organizar la práctica; suponen un cambio de perspectiva, quizás una teoría distinta, pero sobre todo una forma diferente de comunicación pedagógica, una nueva relación con el conocimiento, un desplazamiento del control sobre los procesos de aprendizaje." ... "Se requiere, por tanto, desarrollar instancias mediadoras - centros de transferencia de innovaciones educacionales - que se especialicen en dicha función y la institucionalicen de manera permanente (Brunner, 2000).

Este tiene que ser un proceso profundo, paulatino, general. Muchos autores se refieren ahora a la necesidad de un cambio de paradigma (Braslavsky, 1999). Un modelo alternativo parte del supuesto de que son necesarias escuelas del futuro, en un sistema educativo del futuro, con docentes del futuro, formados con una concepción de futuro. 
La pregunta que surge es como enfrentar desde la formación de los docentes este desafío.

\section{LAS NUEVAS COMPETENCIAS PARA LA ENSEÑANZA}

Lejos de abonar en las posturas que postulan la desaparición de la figura del docente en la nueva educación, creemos que éste es y seguirá siendo irreemplazable en el triángulo didáctico. Pero con la misma fuerza creemos que parte de su profesionalidad se juega en su capacidad, como individuo y como actor social, de redefinir su lugar y su tarea, de adecuarse a nuevos objetivos, de responder a los compromisos de hoy $\mathrm{y}$, fundamentalmente, de poder ser puente adecuado entre una era que termina y un futuro que se abre.

Un repaso de la bibliografía sobre estos temas nos devuelve una mirada pesimista, teniendo en cuenta la perspectiva extremadamente simplista de cómo se abordan estos problemas (Hopkins, 1996). Esto quiere decir que, a pesar de que las experiencias son muchas, las variaciones son pocas. Las líneas desde donde se plantean las soluciones en torno a la docencia, su rol y su formación se pueden resumir en unas cuantas, bien definidas (Martin,1999).

El centro de la cuestión es como pasar de la repetición, con todas las variaciones que esto ha adoptado hasta ahora, a la creación de propuestas diferentes. Ya no alcanza con que un profesor sepa lo que va a enseñar y tenga una buena formación acerca del proceso de enseñanzaaprendizaje. La complejidad de la tarea requiere de una serie de otros elementos más. De ahí que el listado de características deseables en un buen profesor sea cada vez más profuso. Hay entonces una larga tradición que trata de determinar el "perfil" deseable del docente, gracias a lo cual se termina presentando largas listas de cualidades y de conocimientos que las maestras y los profesores deberían tener. Que son, por otro lado imposibles de desarrollar de manera masiva en todos ellos.

Se requiere por ello un cambio de enfoque, una mirada desde otro lado, un cambio de paradigma. Como señala Braslavsky, "se trata de 'reprofesionalizar' a la profesión misma, y no a cada maestro en particular... (y por ello) es posible que la clave para promover la reinvención de la profesión de enseñar consista, nuevamente, en ubicar el foco". En un sistema educativo que debe formar competencias en los alumnos "se trata de que ellos mismos (los profesores) sean competentes y de que tengan una identidad múltiple y consistente" (Braslavsky, 1999).

Intentando resumir ese foco sin caer en el listado de rasgos que conforman un perfil, esta autora opta por pensar las necesidades de formación de los docentes desde una perspectiva alternativa, describiendo dimensiones de la compleja competencia profesional que se requiere 
para poder enseñar. Señala cinco dimensiones fundamentales de la práctica docente: la pedagógico-didáctica, la político-institucional, la productiva, la interactiva, y la especificadora.

\section{La dimensión pedagógico-didáctica}

Una primera dimensión de la competencia profesional docente es sin duda el manejo adecuado de la pedagogía y de la didáctica como base científica de su accionar. Si se piensa la tarea de enseñar como un compromiso de producir resultados buscados, las acciones que se desarrollen para ello requieren un conocimiento de los aspectos profesionales de cómo actuar para lograrlos. Pero, como parte de una competencia, esta dimensión no se resuelve solo a partir de saberes científicos: se trata de integrar el saber académico con el saber de la experiencia ${ }^{1}$. No basta conocer las teorías sobre el aprendizaje o sobre las didácticas especificas del campo de conocimiento que se esta enseñando. A la manera de cualquier otro saber profesional, el marco base de conocimiento es el sustento para la formación de criterios personales - muchos de los cuales se adquieren con la experiencia profesional - que permitan seleccionar la estrategia adecuada entre una serie de estrategias conocidas orientadas a promover los aprendizajes de los alumnos.

Como todo saber profesional, esta competencia reconoce un grado más complejo de se lograr cuando el profesor es capaz de inventar otras estrategias allí donde las disponibles son insuficientes o no pertinentes. En suma, "los profesores que forman sujetos competentes con identidades sólidas conocen, saben seleccionar, utilizar, evaluar, perfeccionar y recrear o crear estrategias de intervención didáctica efectivas y variadas y no se guían por la adhesión a visiones estereotipadas de las teorías pedagógicas de moda...” (Braslavsky, 1999).

\section{La dimensión político-institucional}

Una segunda dimensión de la competencia profesional docente es la que Braslavsky denomina dimensión institucional. Esta aparece en la medida en que la práctica docente no es una profesión liberal. No se ejerce por la propia cuenta y riesgo de quien enseña - como ocurría antes de que se estructuraran los sistemas escolares - sino que ocurre dentro de un ámbito institucional

\footnotetext{
${ }^{1}$ Un profesional no se hace durante sus estudios, sino fundamentalmente cuando empieza a trabajar. Esa instancia de ida y vuelta desde el conocimiento académico a la acción es lo que lo constituye como profesional. En esta idea se basan las concepciones de formación docente continua, que no es lo mismo que más contenidos teóricos. Es claro que no han dado resultado las estrategias de mejorar la formación de los docentes solo alargando la duración de la carrera o pasándola a la universidad.
} 
complejo que articula diferentes instancias, como son el aula, la institución escuela y el sistema educativo.

La dimensión institucional de la competencia profesional docente consiste en la capacidad de articular la macro política referida al conjunto del sistema educativo con la micro política de lo que es necesario programar, llevar adelante y evaluar en las escuelas y en sus espacios mas acotados: las aulas, los patios, los talleres y los ámbitos comunitarios para que la política educativa global pueda tener sus efectos. "Los buenos maestros saben que lo que se decide en los ministerios tiene que ver con lo que pasa en las escuelas y en las aulas pero que, al mismo tiempo, no lo define plenamente. La búsqueda de ampliación de los márgenes de autonomía, los aprendizajes que se adquieren en esa búsqueda y la comprobación de los limites del ejercicio de la propia creatividad permiten por otra parte demandar desde la acción no desde el inmovilismo" (Braslavsky, 1999).

En términos de un nuevo sistema educativo, que sea capaz de cambiar permanentemente, esta dimensión es crucial ya que entender las relaciones entre las políticas generales del sistema educativo, la micro política de la escuela, y el aula permitiría superar las tradicionales posturas enfrentadas frente a las reformas entre los profesores, los estamentos intermedios y los decisores políticos, de manera tal que fuera posible conformar un verdadero espiral creativo.

\section{La dimensión productiva}

Una tercera dimensión es la que Braslavsky denomina productiva, pero que podría también llamarse de comprensión y participación en el mundo actual, ya que se refiere a la necesidad de que todo profesor sea capaz de comprender e intervenir como sujetos en el mundo y como ciudadanos productivos en la política y en la economía actuales. El eje de esta dimensión es la necesidad de que la educación salga de la tradicional postura endogámica que la transforma en un territorio poco grato para quienes deberían beneficiarse fundamentalmente de ella: los alumnos. Esto implica que todo profesor debe conocer y comprender los rasgos centrales de la cultura juvenil, los procesos fundamentales de funcionamiento del sistema económico, político, etc.

Esta cultura endogámica de la mayoría de las escuelas y de las instituciones de formación docente tuvo como consecuencia que estas se alimentaran permanentemente entre sí, sin una fuerte interacción con otras instituciones o ámbitos, sin plantearse preguntas ni formularse respuestas alternativas respecto del mas allá temporal y espacial. Este encierro les ha impedido seguir el ritmo de los cambios en el progreso técnico, en las economías, y en las sociedades. 
"Pero los buenos maestros y profesores suelen ser quienes, pese a eso, siguen interesándose en el mundo y pueden realmente orientar a los niños y a los jóvenes en la identificación de sus raíces y en su proyección hacia el futuro. Esto implica que un desafío fundamental en la reinvención de la profesión del profesor es ampliar el horizonte cultural" (Braslavsky, 1999).

\section{La dimensión interactiva}

La cuarta dimensión de la competencia profesional docente tiene que ver con la calidad de la interacción que es capaz de establecer un profesor. Sabemos que la enseñanza es, por definición, una actividad relacional en la cual lo afectivo juega un papel más que determinante. La dimensión interactiva se refiere a la comprensión y a la empatía con "el otro", que puede ser un alumno, un padre, una madre, un estudiante secundario, una supervisora o los funcionarios de los ministerios, pero también las comunidades en tanto tales, los empresarios, las organizaciones sociales, las iglesias y los partidos políticos. "Los buenos profesores conocen y comprenden la cultura de los niños y los jóvenes, las peculiaridades de las comunidades, las formas de funcionamiento de la sociedad civil y su relación con el Estado. Son capaces de ejercer y promover la tolerancia, la convivencia y la cooperación entre personas diferentes" (Braslavsky, 1999).

Un aspecto a destacar dentro de esta dimensión es la calidad de las relaciones entre los profesores. El hecho de que hasta ahora los profesores se hayan formado para un determinado nivel o modalidad de los sistemas educativos, por un lado, y para una disciplina - en el caso de la educación secundaria - por otro dificulta las relaciones entre ellos y los constituye como en ámbitos separados de trabajo profesional. A esto colabora un modelo institucional que define el trabajo docente como una actividad personal aislada, sin responsabilidades concretas por las cuales responder. Los buenos profesores son capaces de trabajar en equipo con sus pares, de intercambiar sus experiencias críticamente, de modificar permanentemente la tarea, de liderar proyectos.

\section{La dimensión especificadora}

Finalmente, una última dimensión es la llamada especificadora, que es diferente de la de especialización. "La especificación es, según nuestro modo de ver, la capacidad de aplicar un conjunto de conocimientos fundamentales a la comprensión de un tipo de sujetos e institución 
educativa y/o de un conjunto de fenómenos o procesos, complementando los saberes que intervienen en la formación de las dimensiones pedagógico-didáctica, institucional, productiva e interactiva con un mayor dominio de contenidos relacionados con una disciplina o un campo del saber, un conjunto de metodologías o de peculiaridades institucionales."

"La especificación puede realizarse en una disciplina, pero no exclusivamente. También puede tener lugar con respecto a una situación de trabajo - las áreas rurales, por ejemplo - o con otras cuestiones. Por otra parte, no habría que limitar la especificación desde las disciplinas a los profesores de la educación secundaria y la especificación desde otro tipo de conocimientos a los maestros y profesores de la educación inicial o general. Hoy en día parece conveniente que en las escuelas de educación primaria o general haya profesores que pueden especificar su quehacer profesional mediante saberes disciplinarios más sólidos y que en los colegios secundarios existan aquellos que puedan especificar las otras dimensiones a través de saberes más sólidos sobre las instituciones y los sujetos" (Braslavsky, 1999).

Hasta acá lo que serían las dimensiones de la compleja competencia de la docencia profesional, que por supuesto se desarrolla en un sujeto como parte de su crecimiento personal y, como tal forma parte de la realización de cada persona. Por ello, al igual que lo que ocurre en otros campos profesionales y en variadas ocupaciones no profesionales (artísticas, académicas, de negocios, etc.), el desarrollo profesional de cada docente afecta de manera sustantiva la formación de su identidad.

Vale aclarar que también cada una de estas dimensiones comprende espacios complejos y que la concreción de cada una de ellas en una propuesta de formación docente puede abarcar múltiples opciones. Pero, en lugar de ser una debilidad, esto es su fortaleza ya que organiza a partir de pocas categorías conceptuales el cúmulo de requerimientos que habitualmente se incorporan a los perfiles del futuro docente.

Si queremos nuevos docentes es fundamental ser claros en los requerimientos. Los estudios sobre las escuelas eficientes muestran que para serlo limitan sus objetivos y los jerarquizan. Una propuesta de formación docente que pretenda superar a las anteriores pierde fuerza si multiplica sus requerimientos. Resumir los rasgos deseables en un conjunto de dimensiones, entendidas en términos de competencia, permite repensar como formar los nuevos docentes y recrear en cada institución una forma de hacerlo.

Como bien dice Braslavsky, "Se trata de 'reprofesionalizar' a la profesión misma y no sólo a cada maestro en particular"..."se trata de hacernos fuertes en lo que define la profesionalidad 
misma de la docencia y su función social: enseñar para que otros aprendan lo que - sin esta enseñanza - no podrían aprender" (Braslavsky, 1999).

\section{ESCENAS DE LA VIDA COTIDIANA DE UNA INSTITUCIÓN ALTERNATIVA}

Hay suficiente evidencia de que las instituciones formadoras actuales no pueden dar cuenta de una propuesta de este tipo. Su accionar está pensado para las demandas del pasado y no para las competencias del futuro. Si es cierto que todas las escuelas necesitan cambios tenemos que empezar por reconocer que las instituciones formadoras de profesores son las que necesitan los cambios más profundos. Es justamente en este nivel del sistema educativo donde se ha sido menos creativos en torno a la introducción de nuevas prácticas pedagógicas y las propuestas han girado sobre todo en relación con nuevos contenidos pero no en términos de cómo organizar ámbitos ricos en experiencias facilitadoras de aprendizajes alternativos, que generen improntas posibles de ser trasladadas luego a las escuelas.

Creemos que la debilidad estructural de las actuales instituciones de formación docente (sean institutos terciarios o carreras en la universidad) tiene como causa primera el hecho de que su misión es - precisamente - formar profesores porque esa formación está pensada con criterios tradicionales, es decir, pensando que la formación profesional se apoya fundamentalmente en la transmisión de los conocimientos necesarios para enseñar.

Proponemos una redefinición de esta misión. Entendemos que un sistema educativo del siglo XXI debe tener una propuesta diferente pero también debe incorporar mecanismos y procedimientos que le permitan una permanente innovación. Creemos que el lugar donde los docentes aprendan a ser docentes no debe ser un "enseñadero", como son actualmente la gran mayoría de estas instituciones, sino que los profesores deben formarse en lugares en los cuales el motivo primero de su existencia sea la innovación. Centros donde se innove y se ayude a las escuelas a innovar. Centros donde se sea capaz de reconocer las innovaciones que aparecen cotidianamente en las escuelas, y donde se pueda hacer el diagnóstico de cuáles son las necesidades de innovación.

A los efectos de constituir un referente para la discusión, se avanza con una semblanza que permita imaginar cómo sería el funcionamiento de una institución de este tipo, cómo se desarrolla

\footnotetext{
2 Estas "escenas de la vida cotidiana" de estas nuevas instituciones fueron elaboradas por la Lic. Paula Pogré y publicadas en Aguerrondo y Pogré (2001).
} 
la vida cotidiana en estos nuevos centros responsables de la innovación educativa continua. El ejemplo trata de una institución que se ocupa de la innovación (y que forma docentes) en secundaria básica y superior, al mismo tiempo que dedica fuerte cantidad de sus esfuerzos a actividades de re-capacitación y actualización de docentes en servicio, y a la formación para nuevos roles docentes. Acompaña también las tareas de los supervisores. En todos los casos, existen espacios de reflexión y de investigación. Para poder visualizar la propuesta se relatan, a modo de escenas, posibles situaciones. El tiempo: cualquier semana de mayo del año 20??, a cinco años de la creación del centro. (Aguerrondo; Pogré, 2001). Observemos lo que ocurre simultáneamente en diversos espacios de esta institución:

\section{Escena 1}

En uno de los salones, un grupo de docentes y alumnos-futuros docentes, pertenecientes a la carrera de historia, están analizando trabajos de alumnos de $9^{\circ}$ grado de escuelas de la zona guiados por la pregunta ¿Cómo construyen los alumnos la noción de Nación? ¿Pueden diferenciarla de la noción de Estado? ¿Cuáles son los indicadores de esta comprensión? ¿Cuáles son las actividades que les permiten, de un modo más particular, hacer una evaluación diagnóstica continua de estas comprensiones?

Los trabajos son de alumnos de una escuela de la localidad cercana de Puesto Chico donde el Centro está colaborando con los docentes de la secundaria básica desde hace más de un año.

La preocupación inicial de la escuela era la poca posibilidad de transferencia de conceptos que eran capaces de realizar los alumnos con mayores dificultades de aprendizaje, en el área de las ciencias sociales.

Por lo tanto este trabajo no sólo sirve para que quienes se están formando puedan entender cómo se construyen estas comprensiones, sino también para encontrar las estrategias y actividades adecuadas para asesorar a los profesores como trabajar con los niños con mayores dificultades.

\section{Escena 2}

Vía e-mail, un grupo de alumnos-futuros docentes recibe consultas de maestros de escuelas rurales inscriptas en el sitio Directorio de Ayuda del Centro, acerca de cuáles son posibles materiales para usar con sus alumnos de secundaria básica en el área de ciencia y tecnología. Están trabajando los impactos sociales de la potabilización de las aguas. 
Como parte del trabajo de Práctica Profesional 1 todos los alumnos del profesorado, guiados por un profesor tutor, pasan dos horas semanales en este "Consultorio de preguntas y respuestas" a distancia.

Una vez recibida la consulta, el grupo de alumnos-futuros docentes que la recibió es el responsable de ser el "acompañante" de ese maestro o profesor en ejercicio, y de mantener la relación establecida.

Todas las consultas se catalogan y organizan, así como las respuestas y los resultados de las colaboraciones, en una base de datos que es parte de un trabajo de investigación que el Centro está desarrollando acerca de cuáles son las áreas temáticas en que los docentes tienen más dificultades para conseguir material de trabajo, o en que los alumnos en las escuelas tienen más dificultad para aprender.

Con esta base se desarrollan dos proyectos: uno sirve de insumo para el taller de producción de materiales didácticos, el otro consiste en la elaboración de una especie de guía de sitios en Internet donde encontrar información pertinente y ahorrar así tiempos de búsqueda. Estas dos tareas son realizadas por los alumnos-futuros docentes de las diferentes carreras como parte de su plan de formación.

Además de los dos proyectos en marcha, esta base se utiliza con una tercera finalidad. El área de extensión la usa como insumo para la programación de secuencias de seminarios cortos de verano sobre áreas temáticas específicas, invitando especialistas externos cuando es necesario.

\section{Escena 3}

En el aula B, alumnos-futuros docentes de la carrera de Inglés, están viendo la CNN. Discuten luego los posibles usos de la TV por cable en las clases en las escuelas de secundaria básica y superior.

\section{Escena 4}

En la cartelera central del Centro podemos leer:

\section{Hoy a las 20 ATENEO} en el espacio de usos múltiples 
Invitados: directivos y docentes de escuelas de Secundaria Básica y Superior de la región 3 para presentar casos de un mejor aprovechamiento de los tiempos institucionales de profesores y alumnos.

Anotarse en Cátedra de Organización Escolar.

Aparecen además en cartelera:

Los módulos de las carreras de formación que se dictan este cuatrimestre y sus posibilidades horarias.

Un calendario de Conferencias y Debates del Mes.

Un sector de Pasantías Ofrecidas.

Nuevos Sitios en la Web interesantes de explorar

(Es importante recordar que muchos de los módulos previstos para la formación inicial de los alumnos-futuros docentes, son abiertos a todos los docentes del sistema, por lo que los días y horarios de cursada es información que interesa no sólo a los alumnos-futuros docentes del Centro sino a todos los profesores de la provincia).

La cartelera es responsabilidad de los alumnos-futuros docentes, como parte de las tareas de sus tiempos institucionales.

Esta información, además de aparecer en cartelera, se transforma en el protector de pantalla de las computadoras, tanto en las que están ubicadas en los pasillos para uso común como en las que están en las aulas.

Para que todas las escuelas puedan acceder a esta información, es parte de la pagina web el Centro que se actualiza quincenalmente.

\section{Escena 5}

En diferentes espacios los alumnos trabajan independientemente. Estos espacios pueden ser: 
- La biblioteca, donde se puede consultar libros, revistas periódicas, videos o se puede trabajar con la PC de manera individual o con conexión a Internet.

- Los boxes de estudio individual, provistos también con PC conectadas a Internet.

- La cafetería, donde hay mesas que pueden servir para reuniones informales entre los alumnos-futuros docentes, los alumnos-futuros docentes y sus profesores, alumnos-futuros docentes y docentes en servicio, profesores del instituto entre sí, etc.

\section{Escena 6}

En otros espacios, alumnos-futuros docentes están con su profesor en horario de consulta. Cada profesor tiene un box de trabajo individual, donde recibe a sus alumnos, prepara sus clases, avanza en sus investigaciones, escribe sus papers.

\section{Escena 7}

Un grupo de profesores, encargados de la programación de la asistencia a las escuelas de la zona, está evaluando junto con el grupo de supervisores de la zona, los formularios enviados por escuelas pidiendo asistencia. Se evalúan por un lado las necesidades de las escuelas, pero también las innovaciones que ellas realizan para resolver problemas cotidianos. Los supervisores colaboran en la selección de los casos de acuerdo con las prioridades de su plan de supervisión y de las directivas del gobierno central.

Los profesores constituyen grupos de alumnos de diferentes niveles de la carrera que colaboran con ellos en el Itinerario de Asistencia.

El trabajo consiste en concurrir a las escuelas, ayudarlas a completar el diagnóstico del problema, acordar las intervenciones a realizar y acompañarlas en su aplicación.

$\mathrm{Si}$ se trata de innovaciones, el trabajo consiste en describirla, encontrar los marcos teóricos interpretativos, ayudar a la escuela a evaluarla y establecer objetivamente el impacto, y ponerla a disposición de los supervisores y de las demás escuelas para que puedan ser utilizadas en situaciones análogas.

Estas innovaciones forman parte del Directorio de Buenas Prácticas que figura en la página web del Centro.

\section{Escena 8}


Alumnos de una escuela de secundario superior cercana visitan el Centro. Estas visitas responden a varios objetivos:

a) están incluidas en el Programa de aliento a Profesionalización Docente, que busca incentivar el ingreso de alumnos-futuros docentes;

b) permiten a los alumnos de esta escuela "asociarse" al Centro de Recursos y Biblioteca del Centro para consultar materiales disponibles, conociéndolo y viendo la amplia gama de materiales que ofrece, y los modos de consultarlos y/o solicitarlos, así como conocer la posibilidad de consultar material bibliográfico on line;

c) permite difundir e invitarlos a integrar los Círculos de Discusión "la escuela que tengo, la escuela que necesito", que es uno de los componentes de una de las investigaciones que están llevando adelante profesores y alumnos-futuros docentes del Centro como parte del Trayecto “Aproximación a la realidad educativa” y "Construcción de la práctica docente”, en primer año.

\section{Escena 9}

Si abrimos la puerta de algunas de las aulas veremos:

- el desarrollo de clases donde se está trabajando con materiales diversos;

- otras donde un docente está haciendo la presentación inicial de la próxima unidad de trabajo;

- en otras, son los alumnos los que están presentando, ayudándose de medios diversos (PC, videos, esquemas, gráficos), contenidos que vienen trabajando e investigando....

Podríamos seguir describiendo escenas de este día de recorrida por el Centro y encontraríamos docentes del instituto llegando del interior tras una semana de trabajo con un núcleo de escuelas rurales de la provincia, alumnos-futuros docentes relatando sus experiencias en una pasantía que acaban de hacer en una escuela de la provincia con un proyecto innovador, gracias a un convenio de intercambio entre ambos gobiernos provinciales. También encontraríamos una sala donde los alumnos-futuros docentes están rindiendo el examen correspondiente a una de las temáticas de su plan de estudios.

Todas estas actividades no ocurren sin una planificación. Existe un currículo de formación de profesores, que incluye contenidos teóricos y prácticos. Pero no se dan "clases" a la manera tradicional sino que los futuros-profesores estudian por su cuenta y se someten a exámenes periódicamente. Para participar de cada una de las actividades que hemos esbozado deben cumplir con requisitos de conocimientos previos acreditados y su participación, como forma parte 
del plan de formación, requiere de producciones propias que son evaluadas por los profesores y especialistas del Centro. También existen trayectos de capacitación en servicio y de especialización, requisito para el desarrollo profesional continuo y para el avance en la carrera docente.

\section{UNA INSTITUCIÓN HETERODOXA. DIEZ IDEAS-FUERZA PARA PENSARLA}

Como consecuencia de las décadas de intentos de cambios que no han tenido los resultados que se preveía, persisten en nuestros sistemas educativos los viejos problemas, ahora agudizados y más complejos, y han aparecido a su lado nuevas dimensiones y complicaciones, que se transforman en nuevos desafíos. Muchas de las explicaciones y de las soluciones apuntan al profesor. En este marco, podemos decir como Fullan (1993) que "la formación docente tiene el honor de ser, simultáneamente, el peor problema y la mejor solución en educación".

Nuestra hipótesis es que para dar vuelta esta ecuación, es decir, para que los docentes dejen de ser el problema y puedan transformarse en la solución, es menester dar un salto de perspectiva. Se necesitan nuevas escuelas, nuevos docentes. Nuevos docentes para nuevas escuelas, en el marco de un nuevo sistema educativo cuyo compromiso es la formación-para-la-acción (las competencias).

Creemos que ha llegado el momento de animarse a superar las visiones clásicas sobre la formación docente. Se trata de abrir oportunidades para una redefinición del lugar de la enseñanza como actividad social, enmarcándola en una perspectiva realmente profesional. El "ser profesional" docente tiene que ver con la calidad del trabajo que se realiza, el modo y el estilo de conducirse y los estándares que enmarcan esa actividad.

Un punto de partida adicional para re-orientar la formación de los docentes es también la necesidad de superar los fracasos de propuestas que han apostado fundamentalmente a resolver el tema desde más contenidos y/o más tiempo. Estudios empíricos sobre este tema dejan en claro el hecho de que un profesor tenga más conocimientos, ayuda, pero decididamente no lo constituye como buen docente.

En el marco de las ideas anteriores hemos presentado las líneas generales de un modelo heterodoxo de propuesta para la formación docente continua, basado en diez ideas fuerza. 
A. Las primeras dos ideas-fuerza están relacionadas con dos desafíos centrales de la nueva educación.

\section{En una sociedad en permanente cambio es necesario un sistema educativo que contenga mecanismos de cambio permanente}

En un mundo en permanente cambio no alcanza con producir reformas en los sistemas educativos, sino que deben introducirse mecanismos de cambio permanente que no vuelvan obsoletos los cambios. Muchos de los problemas que arrastra nuestra educación son resultado de buenas estrategias introducidas en otros momentos que, con el cambio de las condiciones del contexto, quedan desactualizadas sin que existan mecanismos adecuados para producir los cambios que serian necesarios.

\section{La sociedad del conocimiento propone el uso de los saberes para la acción. Para responder a este desafío se requiere un sistema educativo centrado en la formación de competencias para el hacer}

El paradigma tradicional de la educación centra sus esfuerzos en la transmisión de saberes. Hoy, el énfasis debe ponerse en la formación de competencias complejas, que suponen saberes pero los orientan a la resolución de problemas. En la medida en que el proceso educativo se define por la interrelación entre el docente, el alumno y el conocimiento, reconocer los cambios en la naturaleza de cada uno de ellos y en sus relaciones lleva a necesarias redefiniciones. Por esto, dentro de un nuevo paradigma, ocupa un lugar central la redefinición del rol docente, que puede sintetizarse en la idea del docente transmisor al docente facilitador. El desafío de la formación docente es ¿Cuál es la propuesta adecuada para cumplir con los requerimientos que impone esta nueva visión de un profesor como facilitador del aprendizaje?

B. Para responder a estos dos desafíos proponemos un cambio en el "lugar" de la formación docente dentro del sistema educativo. 


\section{Los profesores no se formarán ni se recapacitarán en instituciones cuyo fin primero sea ese. Serán formados en espacios encargados de resguardar y alentar la innovación del sistema educativo}

El punto de partida inicial que define el cambio de perspectiva es que la propuesta se descentra del objetivo básico de formar docentes. El sentido último es que las instituciones de formación docente sean centros de innovación pedagógica, y su accionar se traduzca en una práctica pedagógica transformada e innovadora que impacte significativamente en el mejoramiento de la calidad de la enseñanza y en los resultados del aprendizaje de todo el sistema educativo.

La diferencia central con lo que conocemos actualmente es que se trata de crear nuevas instituciones formadoras de docentes, que no tengan como misión fundamental formar docentes. Instituciones que cumplan con la tarea de formar ( $\mathrm{y}$ actualizar) docentes pero cuyo fin fundamental sea descubrir, alimentar y difundir la innovación pedagógica, necesaria en el marco de un nuevo sistema educativo.

Centros donde se reflexione sobre los modos de aprender y de enseñar, centros donde diferentes actores puedan realizar experiencias diversas que permitan mejorar permanentemente sus propias propuestas de enseñanza y usarlas como disparadoras de nuevos desafíos.

Se trata, ni más ni menos, de crear dentro del sistema educativo un espacio para la función de R\&D, o sea un espacio para poder retroalimentar la profesión de enseñar para encontrar cada vez mejores maneras de enfrentar y resolver los problemas que se presentan.

Pero no son centros de investigación académica sobre la innovación. Son centros de detección, promoción, difusión, de la innovación permanente a nivel de aula y de institución escolar, para lo cual deben desarrollar actividades de lectura y comprensión de la realidad (investigación) en tanto y en cuanto ésta sirva a su fin principal: generar, sostener y difundir la innovación. Y por esto una de las funciones que deberán cumplir será formar a los profesionales que, en las escuelas, sean los responsables de la puesta en práctica de estas innovaciones, o de generar otras.

De ahí su responsabilidad de formación docente. No como fin en sí mismo; no como tarea aislada del contexto, sino en relación con este nuevo papel que deben jugar en el sistema educativo. 


\section{Un cambio en la visión y en la misión producirá un cambio en los resultados, superando la actual inmovilidad}

Es recurrente en la bibliografía especializada encontrar críticas que demuestran que las propuestas para mejorar la formación de los profesores no superan los marcos clásicos desde donde se la concibió originalmente.

La apuesta en este sentido es generar una propuesta que sea a la vez capaz de resolver las deudas del pasado y de responder a los retos del futuro.

Una novedad es ya crear instituciones encargadas de la innovación permanente pero no en el nivel teórico de las universidades, sino como ayuda directa al hacer de las escuelas y de los supervisores. Pensar la formación y capacitación continua de los profesores en el marco de su propio hacer profesional, agrega valor ya que permite superar la tradicional dicotomía entre teoría y práctica.

C. Las siguientes ideas-fuerza se ocupan de diseñar un modelo de funcionamiento en el cual se pueda contemplar un lugar explícito para cada una de las dimensiones de la competencia de enseñar.

\section{La práctica como centro de la formación de competencias debe provocar cambios en los} modos de enseñar y en la organización de las aulas y de las escuelas

Estas instituciones deben constituirse en un modelo nuevo de institución escolar en el que los futuros docentes hagan la experiencia y tengan la certeza de que es posible un nuevo contexto institucional, que debe ser el modelo para la transformación del resto de las instituciones educativas.

En su compromiso por la formación de competencias (y no solo de saberes), todas las nuevas escuelas deben estar organizadas para "saber hacer", para generar competencias. Por lo tanto, deben tener espacios para el "hacer".

Pasar de la clase frontal a la enseñanza de las competencias supone hacer de la práctica (en cualquiera de los niveles del sistema educativo) el eje del proceso de aprendizaje. Es una nueva concepción de la propuesta de enseñanza que requiere encontrar un modelo didáctico y de organización concreta del aula y de la escuela que supere el esquema tradicional por el cual se enseña la escuela activa desde un discurso y no desde la práctica. 
Se trata de incorporar actividades formativas que avancen hacia una propuesta didáctica que integre realmente la teoría con la práctica.

La actividad principal de estos centros será trabajar con las escuelas, detectando innovaciones pero también ayudando y asistiéndolas para que ellas innoven. En estas tareas trabajarán, en equipo, los "profesores del profesorado" y los alumnos-futuros docentes o profesores en servicio. Como resultado, los futuros docentes aprenderán en el medio de una situación real de asistencia técnico-pedagógica a docentes enfrentados con situaciones cotidianas.

Otro punto interesante es la idea de pasantías institucionales, o "tiempo institucional" de los alumnos-futuros docentes. Superando la concepción más clásica de que las pasantías se hacen fuera de la escuela, se propone utilizar el espacio institucional de estas instituciones para entrenar en el hacer. De este modo, se podrán ofrecer pasantías en diferentes tipos de tareas: administrativas, de asistencia pedagógica, de organización de actividades, de mantenimiento o producción de materiales, etc.

\section{Para ofrecer la oportunidad de un aprendizaje interactivo a lo largo de toda la vida, conviven en las actividades del Centro alumnos-futuros profesores con docentes en servicio en diferentes momentos de su carrera}

La competencia de enseñar se desarrolla durante toda la vida y, como hemos visto, abarca una serie de dimensiones que van más allá de la estricta temática técnico-pedagógica. Por esto, la oferta de formación debe abarcar el amplio marco que va desde la formación inicial a la formación docente continua, pero no como dos instancias separadas, sino como parte de las mismas actividades que se realizan en el Centro.

Se trata de un espacio de formación docente continua (capacitación) de profesores en servicio que se actualizan en la misma escuela, como parte de su trabajo. Entre éstas están los mismos módulos que integran el curriculum de formación. O sea, muchos de los módulos previstos para la formación inicial de los alumnos-futuros-docentes, son abiertos a todos los docentes. Por ello, los días y horarios de cursada es información que interesa no sólo a los alumnos-futuros docentes del Instituto sino a todos los profesores del sistema.

Esto significa que, tanto en las actividades de formación académica, como en las de trabajo para el desarrollo de las competencias, se conformarán equipos de trabajo con profesionales que coordinen y orienten (los equivalentes a los "profesores de los profesorados"), noveles 
interesados en iniciar su formación para el desempeño como profesionales de la enseñanza, y profesores en servicio que estén interesados en crecer profesionalmente.

La propuesta de que "convivan" futuros docentes con docentes en servicio persigue un doble objetivo. Por un lado, se trata de que los futuros docentes tengan la oportunidad de contrastar preguntas e intereses con quienes están ya enseñando. Por el otro, se trata de que quienes se han recibido y están trabajando tengan la oportunidad de volver a rever contenidos académicos para actualizarse y mejorar en sus respuestas frente a los problemas de la enseñanza.

\section{En estos Centros las TICs son parte de los recursos con que se cuenta. No pueden ser pensados sin ellas (aún en contextos de pobreza)}

Independientemente del avance actual en la inclusión de las TICs en las escuelas del sistema, es necesario que los nuevos docentes manejen con comodidad estas herramientas ya que hoy constituyen uno de los lenguajes de la modernidad. Por esto, un desafío importante de estas nuevas instituciones es la necesidad de entrenar a los futuros profesores en el manejo cotidiano de las herramientas informáticas.

Además de la necesaria conexión a Internet y su uso cotidiano por todos los participantes de estos centros, como parte de su tarea, se deben desarrollar actividades formales de asistencia técnico-pedagógica, tutoría y capacitación a distancia de los profesores de las escuelas asistidas.

Esto no formará parte de un programa especial sino que integrará el tipo de tareas comunes que todos los miembros de estos centros estén realizando. ${ }^{3}$ Un desafío fuerte de la nueva educación tiene que ver, precisamente, con la inclusión de las nuevas tecnologías de la información (TICs) en la rutina cotidiana del quehacer educativo.

No son un lujo ni una excepción. Así como no puede pensarse en estos Centros (aún en contextos de pobreza), sin personal docente (formadores de formadores) debidamente titulados y capacitados, sin buena biblioteca, y sin las mínimas condiciones edilicias, tampoco puede aceptarse que no tengan incorporadas rutinariamente las herramientas informáticas básicas.

D. Las siguientes ideas-fuerza se refieren a diferentes aspectos de la organización institucional y del sistema educativo.

\footnotetext{
${ }^{3}$ Se trata de superar las concepciones que agregan al viejo sistema el "aula de computación" y el "profesor de computación”, con lo que esta actividad se aísla y con ello se logra que todos los demás profesores sigan trabajando sin utilizarlas.
} 


\section{Enseñar y aprender más allá de la institución}

Necesitamos escuelas diferentes: escuelas inteligentes. Es decir, escuelas que sean capaces de procesar las demandas de su entorno y de dar las respuestas adecuadas. Y las primeras escuelas inteligentes tienen que ser los lugares donde los docentes se formen. Porque "las escuelas inteligentes deben ser lugares de aprendizaje reflexivo no sólo para los alumnos sino también para los maestros" (Perkins, 1995).

\section{- Tiempo para hacer; tiempo para pensar}

El tiempo para hacer implica por un lado la existencia de diversidad de actividades de aprendizaje, teóricas y prácticas. Esto significa superar la dicotomía entre lo "curricular" y lo "extracurricular" (Torres, 2000). Superar la idea de que los aprendizajes dentro de la escuela deben ser cerrados, rígidos, formales, y que la innovación y el cambio sólo pueden hacerse traspasando las barreras del currículo, más allá de "la escuela".

Por otro lado, la nueva educación necesita un modelo de organización que libere tiempo para pensar. En nuestro modelo de educación occidental, el docente está sometido a un ritmo vertiginoso que no les da tiempo para preparar las lecciones, calificar las pruebas escritas y realizar otras actividades fundamentales de la enseñanza porque todo el tiempo del horario escolar lo pasan dictando clase o paseándose por los pasillos del aula. (Perkins, 1995). Los maestros necesitan tener tiempo fuera de clase durante el horario escolar y dedicarlo a cumplir otras responsabilidades que incluyan el perfeccionamiento de su oficio.

\section{Hacer con otros. La cultura compartida del oficio de enseñar: hacia comunidades de práctica}

La organización de los Centros de Innovación deben permitir pensar en las actividades que se van a emprender, planificarlas, compartir los planes con todo el equipo, aceptar críticas, concurrir a talleres, observar cómo enseñan los colegas, ver videos sobre la práctica de la enseñanza.

Esto supone una conceptualización de la profesión docente como algo que se logra con el esfuerzo. No se "nace" docente: el docente se "hace" gracias al compromiso y a la organización del tiempo, los docentes aprenden a enseñar mejor que lo que su capacidad innata les hubiera pemitido (Perkins, 1995). 
- Colaboración responsable (coaching). Un modelo de aprendizaje para el desarrollo del maestro

El trato que recibe el maestro que acaba de ingresar a la escuela forma parte de la cultura del arte de enseñar. El modelo occidental se sintetiza en la frase “¡Húndase o nade!” A los nuevos maestros se les asigna un aula donde deben enseñar lo mejor posible, con escaso o nulo apoyo por parte de los colegas de más experiencia (Perkins, 1995).

El nuevo modelo no da por sentado que la educación recibida por el maestro hasta el momento de entrar en servicio lo habilita para manejar un aula y dar clase. Los Centros de Innovación, además de ocuparse de la formación profesional inicial, reciben a los profesores en servicio y se organizan de tal forma que los maestros más jóvenes trabajan en equipo con los más experimentados.

Otro rasgo distintivo que aparece es que, dado que su misión central no es solo dar cursos para la formación inicial de docentes sino trabajar para la innovación educativa, es una institución que está abierta permanentemente. Su horario de trabajo es de 8 a.m. a 10 p.m. durante los 365 días del año, desarrollando muchas actividades diferentes, desde asesorías varias (a supervisores, escuelas, grupos de docentes), a instancias de colaboración con universidades, o diferentes cursos de verano. Por supuesto que esto no significa que quienes trabajan en ella no tienen vacaciones, sino que la institución no cierra en período de vacaciones porque este es, precisamente, el momento en que puede ofrecer actividades para los profesores ocupados durante el ciclo lectivo.

\section{Un sistema educativo capaz de organizarse en una red con nodos interrelacionados}

Un sistema educativo en red que, en este caso, define un cambio en la relación entre la institución formadora de docentes y las demás instituciones de enseñanza (horizontal) y recrea una relación diferente con las otras instancias del sistema (servicios de intercambios mutuos).

Los Centros de Innovación se relacionan con las escuelas del sistema no (solo) como espacio para realizar las prácticas de los futuros docentes, sino fundamentalmente como espacio de asistencia técnica y consejo pedagógico de los planteles docentes, a partir de la demanda de las mismas escuelas.

Esto conforma una red que atiende la diversidad ya que una de las preocupaciones centrales de estas nuevas instituciones, en tanto forman parte de un nuevo sistema educativo, es 
construir una pedagogía de la diversidad, o sea encontrar las estrategias adecuadas para enseñar a los alumnos de los sectores más carenciados.

"Cabe pensar que las reformas educacionales del tipo de innovaciones tendrán mayores posibilidades de éxito en la medida en que las escuelas se encuentren integradas en redes que las conecten a otras instituciones, comunidades y personas relevantes que si permanecen aisladas dentro de sus muros... Que un establecimiento escolar y sus miembros estén situados en el entrecruzamiento de muchos flujos - de ideas, de experiencias distintas, de aprendizajes distribuidos, usos y prácticas, modalidades de comunicación e interacción - no puede sino resultar en un clima más favorable para la innovación"... "Por ahora lo importante consiste en diseñar - dentro de las redes existentes - mecanismos que posibiliten la adopción y transferencia de innovaciones aplicadas al proceso de enseñanza y aprendizaje" (Brunner, 2000).

¿De qué se trata, en última instancia? De superar las lógicas clásicas de instituciones cerradas y poco flexibles. De abrirse a las posibilidades de pasar de "ser el problema" a "ser la solución”. En síntesis, instituciones abiertas, desafiantes. Un espacio en el que se abren múltiples posibilidades.

Su objetivo central (ser el responsable y el garante de la innovación pedagógica) permite definir una serie de actividades diversas y no tradicionales que, juntamente con las ya típicas de toda institución responsable de la formación docente, le da un perfil propio que lo diferencia claramente de las imágenes clásicas de nuestras instituciones formadoras.

Se trata de generar instituciones, abiertas, desafiantes. Espacios en los que se abren múltiples posibilidades. Instituciones nuevas, diferentes, que ofrezcan la clase de experiencias que necesita quien debe colaborar con el tremendo desafío que es hoy cambiar la educación.

\section{BIBLIOGRAFÍA}

AGUERRONDO, Inés. <El> Docente frente a la sociedad del conocimiento: elementos para una redefinición de su identidad profesional. In: SEMINARIO IDENTIDAD PROFESIONAL Y DESEMPEÑO DOCENTE EN VENEZUELA Y AMÉRICA LATINA, 2000. Caracas. Anais... Avina, 2001.

. Escuelas del futuro: cómo piensan las escuelas que innovan. Buenos Aires: Papers

Editores; Educación. 2002.

. Formación de docentes para la innovación pedagógica, ponencia. In: SEMINARIO REGIONAL DEL BIE/UNESCO, 2000, Maldonado (Uruguay), Anais... Maldonado, 2001.

$<$ Las> Reformas de la educación en América Latina: análisis de algunos casos nacionales. In: RAMA, G. (Coord.). Mudanças Educacionais na America Latina: situações e condições. Fortaleza: Universidad Federal do Ceará, 1983. p. 13-121. 
AGUERRONDO, Inés; POGRE, Paula <Los $>$ Institutos de formación docente como Centros de Innovación Pedagógica. Buenos Aires: Troquel, 2001.

APPLE, Michael. Markets, standards, teaching and teacher education. Journal of Teaching Education, v. 52, n. 3, May/June 2001.

BRASLAVSKY, Cecilia. Re-haciendo escuelas: hacia un nuevo paradigma de la educación latinoamericana. Buenos Aires: Santillana, 2000.

BRUNNER, José Joaquín. Educación: escenarios de futuro: nuevas tecnologías y sociedad de la información. Santiago de Chile: PREAL, 2000. (Documento, 16).

BURROUGHS, Robert. Composing standards and composing teachers: the problem of National Board Certification. Journal of Teaching Education, v. 52, n. 3, May/June 2001.

FULLAN, Michel. Change forces. Londres: Palmer, 1993.

GAJARDO, Marcela. 10 años de reformas educativas en América Latina. Santiago de Chile: PREAL, 1999. (Documento, 14).

Reformas educativas en América Latina: balance de una década. Santiago de Chile:

PREAL, 1999. (Documento, 15).

HOPKINS, David. New rules for the radical reform of teacher education. In: HUDSON, Andy;

LAMBERT, David (Ed.). Exploring futures in initial teacher education: changing key for changing times. London: Bedford Way Papers, 1996.

MARTIN, Michaela. $<$ The $>$ Role of the university in initial teacher training: trends, current problems and strategies for improvement. Paris: IIEP/Unesco, 1999. (Contributions, 32).

MATUS, Carlos. Planificación de situaciones. Caracas: CENDES, 1976.

OSZLAK, Oscar. Quemar las naves o cómo lograr reformas estatales irreversibles. Aportes para el Estado y la administración gubernamental, v. 6, n. 14, Buenos Aires, primavera 1999.

PERKINS, David. $<$ La $>$ Escuela inteligente: del adiestramiento de la memoria a la educación de la mente. Barcelona: Gedisa, 1995.

PERRONE, Vito. ¿Cómo podemos preparar nuevos docentes? In: STONE WISKE, Martha (Comp.). Enseñanza para la comprensión: vinculación entre la investigación y la práctica. Buenos Aires: Paidós, 1999.

SCHIEFELBEIN, Ernesto. Relación entre la calidad de la educación y el modelo de enseñanza frontal en América Latina. Boletín del Proyecto Principal de Educación en América Latina y el Caribe, n. 29, 1992.

SHÖN, D. A. <La> Formación de profesionales reflexivos. Madrid: Paidós, 1992.

SUAREZ, Francisco M. et al. Analista organizacional: ¿profesión o saber? Buenos Aires: Macchi, 1999.

TEDESCO, Juan Carlos. <El> Nuevo pacto educativo. Madrid: Grupo Anaya SA, 1995.

TISHMAN, Shari; PERKINS, David; JAY, Eileen. Un aula para pensar: aprender y enseñar en una cultura de pensamiento. Buenos Aires: Aique Grupo, 1994.

TORRES, Rosa María. Itinerarios por la educación latinoamericana: cuaderno de viajes.

Buenos Aires: Andrés Bello; Paidós, 2000.

Recebido em: setembro 2009

Aprovado para publicação em: outubro 2009 